\title{
Mechanisms and sites of ultra high energy cosmic ray origin
}

\author{
M. Ostrowski \\ Obserwatorium Astronomiczne, Uniwersytet Jagielloński, \\ ul. Orla 171, 30-244 Kraków, Poland (e-mail: mio@oa.uj.edu.pl)
}

\begin{abstract}
.
We shortly discuss several astrophysical scenarios leading to cosmic ray acceleration up to extremely high energies reaching the scale of $10^{20} \mathrm{eV}$. The processes suggested in the literature include acceleration at relativistic jet terminal shocks and shear boundary layers, shocks in large scale accretion flows onto supergalactic cosmic structures, particle reflections from ultra-relativistic shocks postulated to exist in sources of gamma ray bursts, the processes involving the neutron star rotating magnetospheres and dormant quasars. Some of these objects can explain cosmic rays with highest energies if one tunes the model parameters to limits enabling the highest acceleration efficiency. We also note that some of the considered processes allow for acceleration efficiency in the Hillas diagram, $\beta$, to be much larger than unity. The present paper is based on a review talk presented during the European Cosmic Ray Symposium in Lodz (2000).
\end{abstract}

\section{Introduction}

Present search for explanation of the observed ultra high energy (UHE) cosmic rays (CRs) resembles somewhat a random walk among possible and impossible particle acceleration models and wide range of more or less exotic physical concepts and particles. The expanding space of such studies is described by more and more numerous review papers appearing in ASTRO-PH listings. The present one is limited to discussing some astrophysical mechanisms proposed to accelerate cosmic rays up to the highest observed energies. In our opinion necessity to seriously consider sources involving 'strange' particles or new physics will come after astrophysical models applying the classical physics are rejected by observations. A review of UHE CR observations is presented by e.g. Bertou et al. [5].

The factors limiting energies of particles accelerated in astrophysical sources include an acceleration rate and a loss rate due to synchrotron radiation and IC or inelastic scattering. However, for the highest considered energies quite often an upper energy limit is provided by a finite source extent. To evaluate the role of this last factor one needs information about magnetic fields present within its volumes. For objects with relatively strong magnetic fields such data are quite often firmly constrained by 
radio and high energy observations. Contrary to that, knowledge of weak magnetic fields in extragalactic space or in galactic coronae, important for the discussed below models involving supergalactic accretion shocks or local sources concentrated toward the galaxy centre, is only fragmentary. Therefore, let us start with a short review of the new evidence on such magnetic fields, which could also substantially influence transport of $\sim 10^{20} \mathrm{eV}$ particles reaching Earth. Next, we will discuss a few astrophysical 'bottom-up' models for generation of such particles. Our intention is not to discuss the full literature of the subject, but rather to overview a set of characteristic publications presenting the discussed models.

Below, in most cases we use a term 'particle' for protons.

\section{Extragalactic magnetic fields}

The importance of any considered magnetized structure for particle acceleration can be evaluated by comparing its size to the particle gyroradius团

$$
r_{g} \approx \frac{1}{Z} \frac{E_{18}}{B_{-6}} \quad \mathrm{kpc}
$$

where $Z$ is a given nucleus charge state. Early evaluations of the field strength at largest scales yielded usually upper limits only of the magnitude order $10^{-9} \mathrm{G}$. The direct observations of extended extragalactic synchrotron glows started in 1990, and were continued in last few years, revealing much stronger fields within galaxy clusters and even in the intracluster space (for review see Kronberg [13]). Estimates show, that $B$ may reach $\sim 1 \mu \mathrm{G}$ in rich clusters and approximately $\sim 0.1 \mu \mathrm{G}$ in supergalactic accretion flows and in supergalactic structures at several Mpc scales. Turning to smaller scales a series of spiral galaxy observations proves existence of extended - with the vertical scale $\sim$ several kpc - magnetic coronae near some (not all) galaxies with a few $\mu \mathrm{G}$ magnetic fields (cf. Beck [2]). Even irregular galaxies show well organized magnetic field structures (Chyży et al. [8]). Growing evidence supporting the Kronberg's claim, that magnetic fields appear to be all-pervasive in space, weaken the opinion that the UHE cosmic ray showers' directions point approximately to sources of the observed particles (cf. Sigl et al. [18]). However, there exists also a growing number of observed particle pairs and triplets above a few times $10^{19} \mathrm{eV}$ which could suggest the opposite (cf. Uchihori et al. [20]).

\section{Particle acceleration in relativistic jets}

Relativistic jets occurring in FRII radio galaxies carry large energy up to the radio 'hot spots' situated far $(\sim 100 \mathrm{kpc})$ from the central source. These hot spots are

$\ddagger$ We often use the notation of the form $X_{Y}$ for a quantity $X$ divided by $10^{Y}$ of the respective units, e.g. for energy $E_{18} \equiv E / 10^{18} \mathrm{eV}$, for the magnetic field $B_{-6} \equiv B / 10^{-6} \mathrm{G}$, or for a distance $R_{6} \equiv R / 10^{6}$ $\mathrm{cm}$. 
believed to harbor strong, mildly relativistic shocks dissipating the jet bulk kinetic energy into heating plasma, generating magnetic fields and efficiently accelerating energetic particles. Because of relatively slow radiative losses for protons such shocks are prospectus UHE cosmic ray accelerators. Additionally, an active role in accelerating UHE cosmic rays can play a velocity shear layer at the relativistic jet side boundary. Let us discuss these possibilities in some detail.

\subsection{Relativistic shocks in hot spots of FRII radio galaxies}

A full elaborated model of UHE CRs acceleration at the relativistic jet terminal shock was presented by, e.g., Rachen \& Biermann [17]. They considered a shock wave with parameters derived from observations of hot spots in the considered sources. One can model the downstream region of the shock to be a circular slice of compressed plasma with the radius in the range $R \approx 0.3-3 \mathrm{kpc}$, thickness $H \approx 0.1-2 \mathrm{kpc}$, the magnetic field $B \approx 0.4-0.6 \mathrm{mG}$. The evaluated jet velocities are in the mildly-relativistic range $u_{j} \approx 0.2-0.5$ c. In order to derive the upper limit for the accelerated proton energy, $E_{\max }$, we compare the shock acceleration time scale given by Rachen \& Biermann for a parallel ' $\|$ ' shock

$$
\tau_{a c c}=\frac{20 \kappa_{\|}}{u_{j}^{2}},
$$

where $\kappa_{\|}$is the cosmic ray diffusion coefficient parallel to the mean magnetic field, with the loss time scale scaled to that of the synchrotron radiation

$$
\tau_{\text {loss }}=\frac{C}{B^{2}(1+X) \gamma_{p}},
$$

where $C\left(\approx 5 \cdot 10^{24}\right.$ s for $B$ given in $\left.\mathrm{mG}\right)$ is a constant, $X$ represents (in some conditions a large, varying with the local conditions and particle energy) correction to $\tau_{\text {loss }}$ due to inverse-Compton (' $\mathrm{IC}$ ') and inelastic collisions, and $\gamma_{p}$ is a proton Lorentz factor. Near the shock, Rachen \& Biermann [17] considered the non-linear Kolmogorov turbulence extended up to the scales comparable to the hot spot size. Thus, in the long wave range important for the highest energy particle scattering the diffusion was the Bohm diffusion, $\kappa_{\|} \approx \frac{1}{3} r_{g} c$, leading to the most rapid acceleration. Then, for 'typical' hot-spot parameters $B=0.5 \mathrm{mG}, u_{j}=0.3 \mathrm{c}, R>H \approx 1 \mathrm{kpc}, X<1$, the conditions $\tau_{\text {acc }}<\tau_{\text {loss }}$ and $r_{g}<H$ can be satisfied up to energies of a few $10^{20} \mathrm{eV}$.

However, some of the above assumptions or evaluations are only rough estimates,

which make the derived $E_{\max }$ somewhat uncertain. For example a required diffusive size for $\sim 10^{20} \mathrm{eV}$ particles seems to be at least (and in fact more than) $\left(c / u_{j}\right) r_{g}$ (cf. Drury [9]), otherwise the particle spectrum exhibits a cut-off (see also below). Also, the turbulence structure downstream of the shock can substantially deviate from the assumed Kolmogorov form. 


\subsection{Acceleration at the jet shear boundary layer}

Ostrowski $([14,15])$ discussed the process of particle acceleration up to ultra high energies at tangential velocity transitions at side boundaries of relativistic jets. An UHE particle can cross such boundary to inside or to outside the jet, then be scattered back to cross the jet boundary again. If the process repeats, each boundary crossing increases particle energy by, on average,

$$
\frac{\Delta E}{E}=\eta_{E}(\Gamma-1)
$$

where $\Gamma$ is the jet Lorentz factor and the efficiency factor $\eta_{E}$ depends on particle anisotropy at the boundary and, thus, on the character of MHD turbulence responsible for particle scattering. For highly turbulent conditions $\eta_{E}$ can be a substantial fraction of unity, decreasing like $\sim \Gamma^{-1}$ for larger $\Gamma$. As a result high energy particles near the jet boundary can be accelerated forming a power-law spectrum up to some cutoff energy, $E_{c}$, appearing when the respective particle gyroradius $r_{g}\left(E_{c}\right)$ becomes comparable to the jet radius, $R_{j}$. The performed simulations show that $E_{\text {max }}$ obtained in the boundary acceleration process is comparable to the discussed above maximum particle energy obtained at the terminal shock. It grows with increasing $u_{j}$, but always $r_{g}\left(E_{c}\right)<R_{j}$ : in the simulations for mildly relativistic jets usually $r_{g}\left(E_{c}\right)<0.1 R_{j}$. An additional interesting feature of this acceleration process is the fact, that particles escaping diffusively from the jet vicinity can posses an extremely hard spectrum, with the power concentrated in particles near $E_{c}$.

The analogous acceleration processes can act at ultrarelativistic jets in blazars (Ostrowski [15]). However, due to expected decrease of acceleration efficiency with particle anisotropy, $\eta_{E} \propto \gamma^{-1}$, the highest energies are still limited by the geometric condition $r_{g}\left(E_{c}\right)<R_{j}$. For characteristic conditions derived for such jets, $B \sim 1 \mathrm{G}$ and $R_{j} \sim 10^{16} \mathrm{~cm}$, particle energies above $10^{19} \mathrm{eV}$ can be obtained. When considered as the source of UHE CRs escaping into the intergalactic space such processes can be further degraded by particle interactions with the strong ambient photon field and/or the diffuse medium near the active galactic nucleus in the parent galaxy. Till now these possibilities were not discussed in detail.

\section{Compressive flows onto supergalactic structures}

Hydrodynamic modelling of cosmological structure formation yields extended flat or cylindrical supergalactic structures of compressed matter, including galaxy clusters as its sub-components. Diffuse plasma accreted with velocities $u \sim 10^{3} \mathrm{~km} / \mathrm{s}$ at such extended at several Mpc structures can form large scale shocks seen in radio observations

mentioned in section 2. Considering UHE particle energization at such shocks (e.g. Kang et al. [12]) in the process of Fermi diffusive acceleration meets however serious obstacles. In the considered acceleration region with $\sim 0.1 \mu \mathrm{G}$ magnetic field the $10^{20} \mathrm{eV}$ proton posses a gyroradius $r_{g} \sim 1 \mathrm{Mpc}$. Thus (cf. Drury [9]), the particle mean free path 
$\lambda>r_{g}$ leads to the unreasonably large diffusive region required for acceleration, with the size $L_{\text {diff }}>\frac{c}{u} \lambda \sim 300 \mathrm{Mpc}($ !), and the acceleration time comparable to the age of the universe. As the considered mean free path is the one normal to the shock surface, $\lambda_{n}$, the above authors considered the so called 'Jokipii diffusion' regime at perpendicular shocks (i.e. where the shock normal is perpendicular to the mean magnetic field), with $\lambda_{n} \ll r_{g}$. Such models formally allow for larger energies of accelerated particles, but can increase the upper energy limit in comparison to parallel shocks at most by a factor of a few (if at all, cf. [16] ). It is due to the fact that the Jokipii diffusion requires a medium amplitude perturbations of the magnetic field, and thus large mean free paths, $\lambda_{\|} \gg r_{g}$, or drifts along the considered shock structure.

In some way analogous (super)galactic scale shock can be formed and accelerate particles in the observed cases of colliding galaxies (Al-Dargazelli et al. [1]). However, in analogy to the discussed above constraints the evaluation of the upper particle energy limit presented by these authors seems to be over optimistic.

\section{Rotating neutron star magnetospheres}

Extremely powerful cosmic engines are provided by rapidly rotating neutron stars. Of particular interest are the objects with magnetar-like magnetic fields $\left(B \gg 10^{12} \mathrm{G}\right)$ and rotation periods, $T$, in the mili-second range.

\subsection{Strong magnetic field region}

A rotating magnetosphere generates an electric field of the magnitude $\sim \frac{1}{c}|(\boldsymbol{\Omega} \times \mathbf{R}) \times \mathbf{B}|$. Multiplied by the stellar radius $R$ this field translates into a voltage $\Delta \Phi \sim$ $10^{20} \mathrm{~V} B_{13} R_{6}^{2} / T_{-3}$, which could be used for acceleration of charged nuclei. However, the $\left(e^{+}, e^{-}\right)$pairs created in the pulsar magnetosphere will short circuit the above potential drop to values a few orders of magnitude lower (cf. Venkatesan et al. [22]). Additionally, a more realistic geometry would introduce a further decrease of $\Delta \Phi$ by a factor $\sim R \Omega / c \sim 0.1$. If one takes into account a possible catastrophic energy changes in the strong magnetic field and the radiation field near the pulsar, the co-rotating field region of the pulsar magnetosphere does not seem to be a possible site for extremely energetic nuclei acceleration (only iron or some other heavy nuclei, probably the not fully ionized ones, can be available there). Venkatesan et al. [22] expect that the far field region, near the outer gap or even behind the light cylinder, can be a more promising acceleration site for UHE particles. However, no detailed model of such process was presented till now.

\subsection{Ultrarelativistic wind zone and its terminal shock}

As discussed by numerous authors the rapidly rotating magnetized neutron stars can be sources of ultra-relativistic winds, as (probably) seen in the centre of the Crab nebula. If iron nuclei can be injected from the star surface into the magnetosphere, then they 
start to co-move with the forming wind, reaching its terminal velocity. As discussed by Blasi et al. [6] for newly born neutron stars with $T<10 \mathrm{~ms}$ and $B \sim 10^{12}-10^{14} \mathrm{G}$ the wind terminal Lorentz factor can reach $\Gamma_{\text {wind }} \sim 10^{9}$. Thus the iron nuclei carried with the wind have in the 'laboratory' frame typical energies $\approx 4 \cdot 10^{20} B_{13} \Omega_{3}^{2} \mathrm{eV}$. At the wind terminal shock the nuclei are injected into the interstellar medium with these energies. For sources distributed in our Galaxy very small source efficiency is sufficient to explain particle flux observed at Earth. Of course, if such processes provide UHE CRs, an anisotropy toward the Galactic centre should be observed. However, as claim the above authors, if our Galaxy posses an extended magnetized corona the anisotropy at the northern hemisphere pointing outside the Galactic Centre could be quite small at $10^{20} \mathrm{eV}$ (?). One may note that the presented model contradicts interpretation of the Fly Eye result suggesting transition to lighter cosmic ray composition at highest energies. Also efficiency of iron ions pull out from the neutron star surface is a theoretically undetermined quantity yet.

\section{6. 'Dead quasars'}

Observations show a much higher density of quasars in the early universe, at cosmological redshifts $z \approx 2$, than today. Thus a number of present day galaxies must harbour supermassive black holes in their centres, some of them rapidly rotating. Only an extremely low accretion rate due to lack of freshly acquired gas near such black holes makes them to be inactive 'dead quasars', without any clear radiative or jet observational signature. Nevertheless, even a small accretion rate can bring magnetized plasma toward a rotating black hole and to form a stressed magnetosphere near to it. Boldt \& Ghosh [7] estimate the magnetic field near the black hole horizon of a radius $R$, to reach $B \sim 10^{4} \mathrm{G}$ and to generate an effective electromotive force $\sim c B R \approx 4.4 \cdot 10^{20} B_{4} M_{9} \mathrm{~V}$ accelerating nuclei (a black hole mass $M_{9} \equiv M / 10^{9} \mathrm{M}_{o}$ ). This force could accelerate the observed UHE cosmic rays in nuclei of nearby inactive massive galaxies. However, an ability of the small amount of accreted plasma to keep the black hole magnetic field at $\sim 10^{4} \mathrm{G}$ is not proved and should be discussed quantitatively.

\section{Ultrarelativistic shock waves in GRBs}

The observed approximately once a day gamma ray bursts are believed to originate from ultra-relativistic shocks, with the Lorentz $\Gamma$ factors reaching values $\sim 10^{3}$. Basing on an oversimplified acceleration model Vietri [21] and Waxman [23] suggested that such shocks could provide UHE CRs also. Later discussions by Gallant \& Achterberg [10] and Bednarz \& Ostrowski [3] show that due to extreme particle anisotropy (upstream of the shock the energetic particle distribution has an opening angle $\sim \Gamma^{-1}$ ) the acceleration process is gradual, with $\Delta E / E \sim 1$, and the considered scenarios for GRB shock acceleration lead to particle energies up to $\sim 10^{18} \mathrm{eV}$. The situation could be more promising if the shock propagates in the region of strong magnetic field, like in the pulsar 
wind zone. However no one attempted to model such acceleration process including into consideration the pulsar wind velocity field with a possible large Lorentz factor and the sector-like magnetic field structure. The process of particle reflection from the shock leading in principle to fast acceleration with $\Delta E / E \sim \Gamma^{2}$ is much less efficient than thought previously (Bednarz \& Ostrowski [4]). Also Stecker [19] pointed out that GRBs, possibly following the star formation rate, are much less frequent in the local universe than in the young galaxies at redshifts above 1. He evaluates that particles from such local unatenuated sources can explain only a small part of the observed particle flux at highest energies. Thus, in our opinion, it is highly questionable if GRBs could generate the observed highest energy cosmic rays.

\section{Concluding remarks}

The Hillas [11] diagram presents possible astrophysical sources of ultra high energy particles on the plane ' $B$ versus $L$ ' ( $L$ - linear size of the source), where the regions of $r_{g}<\beta L$ roughly define the allowed range for sources with the 'acceleration parameter' $\beta$ (Fig. 1). Hillas introduced $\beta$ as the respective velocity parameter for the studied acceleration mechanisms. Traditional objects placed on the diagram include galaxy clusters, AGNs, FRII radio sources and pulsars. Recent particle acceleration models make the Hillas diagram more densely populated due to taking into consideration objects like 'magnetars', 'dead quasars', or ultrarelativistic pulsar winds and GRB shocks. They also require a more general meaning for the parameter $\beta$. Traditional approach considers $\beta$ to be comparable or smaller than unity. For example particle acceleration at relativistic shock can be characterized with $\beta \approx 1$, while the non-relativistic shock propagating with velocity $u$ yields at most $\beta \sim u / c$. In the last expression the factor $u / c$ comes due to the fact that the source size must be greater than the required diffusive length scale $\sim r_{g} c / u$ for highly turbulent conditions (cf. [9]). The processes like a particle reflection from an ultra-relativistic shock or a bulk flow acceleration on the cost of the pulsar wind Poynting flux introduce a 'new dimension' into the above efficiency considerations. The model of Blasi et al. [6] considers particles resting with respect to the pulsar wind and introduces high energy particles when injected at the wind terminal shock. The model works well even with zero energy particles in the wind rest frame and - in this frame - the condition $r_{g}<\beta L$ does not provide any limit to particle energy in the 'external observer' rest frame. In the model involving acceleration by cosmic ray reflections from ultra-relativistic shocks of GRBs the condition $r_{g} \sim L$ downstream the shock (within the source) yields $r_{g} \sim \Gamma L$ after the reflection, and thus $\beta \sim \Gamma \gg 1$ (cf. Appendix).

Ability to accelerate particles up to ultra high energies $\sim 10^{20} \mathrm{eV}$ by sources (models) discussed shortly in this paper (cf. Table 1) and a few further ones appearing in the literature is an open problem. Very few of these propositions reached even a semiquantitative level, involving a detailed modelling of individual particle acceleration. Quite often for the evaluated process one assumes the physical conditions providing 


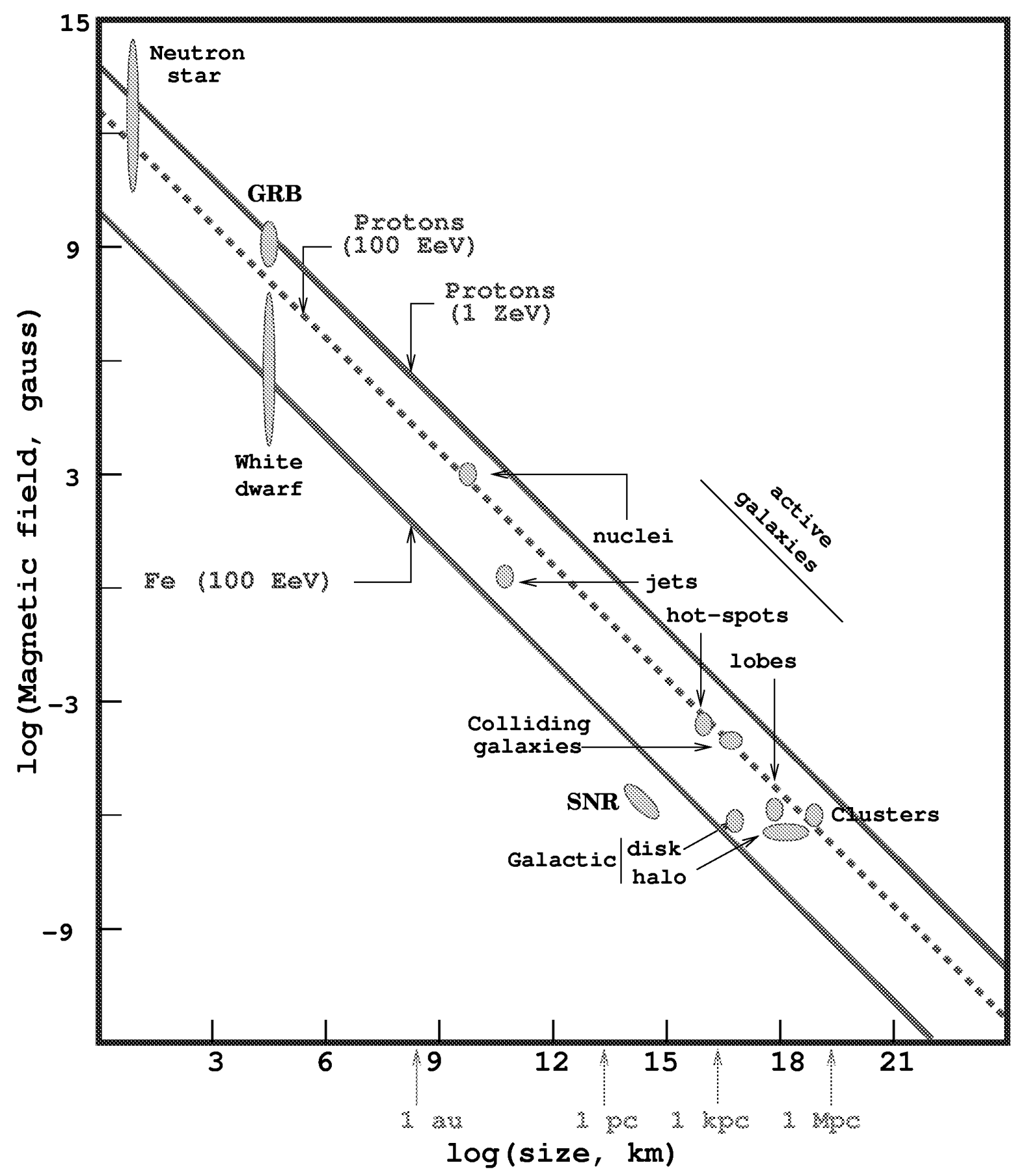

Figure 1. The Hillas diagram. Acceleration of cosmic rays up to a given energy requires conditions above the respective line. Placement of an oval for GRB sources is somewhat controversial at such diagram, as discussed in the Appendix. The figure was kindly provided by Murat Boratav. 


\begin{tabular}{|c|c|c|c|c|c|c|}
\hline & Acceleration site & $\begin{array}{l}\text { Expression for } \\
\qquad E_{\max }\end{array}$ & $\mathrm{p} / \mathrm{O} / \mathrm{Fe}$ & $\mathrm{E}_{\max }$ & $\begin{array}{l}\text { Possible reducing } \\
\text { factors }\end{array}$ & $\begin{array}{l}\mathrm{E}_{\max }- \\
\text { guess }\end{array}$ \\
\hline 1 & $\begin{array}{r}\text { Relativistic jets: } \\
\text { - terminal shock } \\
\text { - side boundary }\end{array}$ & $\begin{array}{l}r_{g}<H \\
r_{g}<R_{j}\end{array}$ & $\begin{array}{l}\mathrm{p} \\
\mathrm{p}\end{array}$ & $\begin{array}{l}>10^{20} \\
>10^{20}\end{array}$ & realistic B structure & $\begin{array}{l}\leq 10^{18} \\
\leq 10^{19}\end{array}$ \\
\hline 2 & $\begin{array}{c}\text { Supergalactic } \\
\text { accretion shocks }\end{array}$ & $\mathrm{r}_{\mathrm{g}} \leq 1 \mathrm{Mpc}$ & $\mathrm{p}$ & $>10^{19}$ & particle escape & $\sim 10^{17}$ \\
\hline 3 & $\begin{array}{l}\text { Rotating neutron stars: } \\
\text { - region of strong } \mathbf{B} \\
\text { - wind and shock }\end{array}$ & $\begin{array}{c}10^{20} \mathrm{Z} \mathrm{B}_{13} \mathrm{R}_{6}^{2} / \mathrm{T}_{-3} \\
4 \cdot 10^{20} \mathrm{~B}_{13} \Omega_{3}^{2}\end{array}$ & $\begin{array}{l}\mathrm{Fe} \\
\mathrm{Fe}\end{array}$ & $\begin{array}{l}>10^{20} \\
>10^{20}\end{array}$ & $\begin{array}{l}\text { geometric factors, } \\
\text { pairs, ... } \\
\text { injection efficiency }\end{array}$ & $\begin{array}{c}? \\
>10^{17}\end{array}$ \\
\hline 4 & "Dead quasars" & $4 \cdot 10^{20} \mathrm{Z} \mathrm{B}_{4} \mathrm{M}_{9}$ & $\begin{array}{l}\mathrm{p}, \mathrm{O} \\
\mathrm{Fe}\end{array}$ & $>10^{21}$ & $\begin{array}{c}\text { details of physical } \\
\text { process }\end{array}$ & $\begin{array}{l}<10^{17}, \\
<10^{21}\end{array}$ \\
\hline 5 & GRB shocks & $\mathrm{r}_{\mathrm{g}}<\Gamma \mathrm{L}$ & $\mathrm{p}, \mathrm{Fe}$ & $>10^{20}$ & B structure & $\begin{array}{l}<10^{18}, \\
<10^{14}\end{array}$ \\
\hline 6 & $\begin{array}{l}\text { Other, not discussed } \\
\text { here (cf. Fig. } 1 \text { and } \\
\text { current literature) }\end{array}$ & $\begin{aligned} \mathrm{r}_{\mathrm{g}} & <\mathrm{L}(?) \\
\mathrm{r}_{\mathrm{g}} & <\Gamma \mathrm{L}\end{aligned}$ & $\begin{array}{l}\mathrm{p} \\
\mathrm{O} \\
\mathrm{Fe}\end{array}$ & $<10^{21}$ & $\begin{array}{l}\text { realistic perturbed } \mathbf{B} \\
\text { and velocity } \\
\text { structure }\end{array}$ & $<10^{21}$ \\
\hline
\end{tabular}

Table 1. A list of UHE CR acceleration sites discussed in the text. In the third column we list main, order of magnitude conditions for the maximum energy of accelerated particles, $E_{\max }$. In the next column the considered nuclei are mentioned; here $\mathrm{O}$ stands for all medium atomic number nuclei. In the last column the author's estimates of $E_{\max }$ are provided, taking into account his subjective evaluation of various reducing factors limiting the acceleration process.

the most efficient acceleration, these conditions are usually only weakly constrained by observations. Besides these reservations one should note a growing number of 'bottom-up' models, using a classical physics to explain UHE CR origin. Possibly one of these or a new proposed mechanism will prove to work. However, the main present day task for UHE CR astrophysics is to increase the number and quality of observational data, to allow for a more serious critical evaluation of the considered acceleration models and to discuss transport processes and forming the observed particle spectrum. In particular, besides the traditional questions about UHE cosmic rays (spectrum, anisotropy, what particles we observe), a number of astrophysical issues must be resolved like understanding of the intergalactic magnetic field structure, confirmation 
or not of the ultrarelativistic winds from pulsars, existence or not of the galactic winds, direct detection and/or explaining composition of the cosmic dark matter, etc. When considering chances for understanding UHE cosmic rays one have to take into account the fact that we are still far from explaining mechanisms providing much better studied lower energy cosmic rays.

\section{Acknowledgements}

I am grateful to Grażyna Siemieniec-Oziębło, Marek Sikora, Włodek Bednarek and Heniek Wilczyński for discussions on UHE CRs, for Maria Giller for critical remarks on the manuscript, and for Murat Boratav for providing the Hillas plot. Remarks of the anonymous referee helped to improve the final version of the paper. The work was supported by the Komitet Badań Naukowych within the project 2 P03B 11217 and the grant PB 258/P03/99/17.

\section{Appendix}

To draw a version of the Hillas plot presented on Fig. 2 we consider the situation with the magnetic field energy density being in equipartition with the plasma energy density upstream of the shock: the sound velocity in the upstream plasma is assumed $v_{s}=30$ $\mathrm{km} / \mathrm{s}$ and the respective plasma density is defined by the equipartition condition. One should note that the magnetic field downstream of the shock, $B^{\prime}$, is much stronger than the upstream value given at the horizontal axis. The compressed ('c') upstream field reaches a downstream value $\sim 3 \Gamma$ higher, while in the often considered case of the equipartition ('e') between the magnetic field and the plasma downstream of the shock the required field is higher by an additional large factor $\sim c / v_{s}$. The curves presented at Fig. 2 - solid lines for protons and dashed lines for Iron nuclei - are plotted for particles reaching $10^{20} \mathrm{eV}$ after reflection from the shock, as measured upstream of the shock. To draw the figure each value of the upstream magnetic field given at the $\mathrm{x}$-axis was transformed downstream the shock according to the ' $c$ ' or ' $\mathrm{e}$ ' prescription and a gyroradius $r_{g}\left(B^{\prime}\right)$ (Eq. 1) was derived for a particle with the energy $10^{20} \mathrm{eV} / \Gamma$ and the transformed magnetic field $B^{\prime}$. This value $r_{g}\left(B^{\prime}\right)=L$ is given at the y-axis. One should note that the size $L$ has to be a distance between causally connected regions along the shock. For example for the spherical shock with a radius $R$ the condition $L<R / \Gamma$ has

to be satisfied. On the figure, we do not present results for protons with the radiative loss time scale (Eq. 3) downstream of the shock shorter than the derived $L / c$. For a reference at the upper right corner of each panel a dotted line is added representing the $\beta=1$ line for $10^{20} \mathrm{eV}$ protons at the original Hillas plot: $r_{g}(B)=L$. 


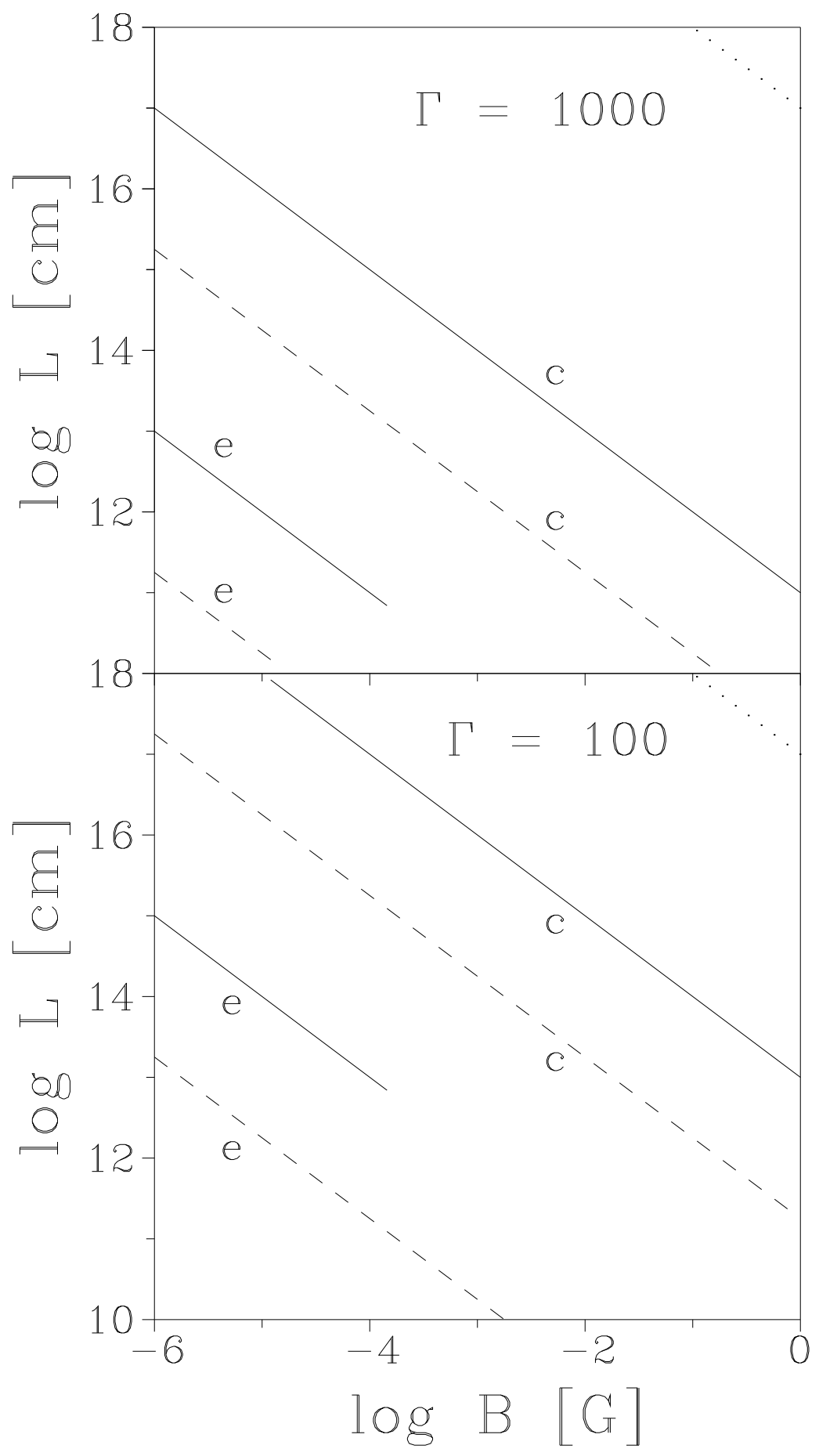

Figure 2. A version of the Hillas plot illustrating the possibility of particle acceleration up to $10^{20} \mathrm{eV}$ by reflection from the ultra-relativistic shock with the Lorentz factor $\Gamma=10^{3}$ or $10^{2}$. At the axes we present the magnetic field, $B$, upstream of the shock (as measured in the upstream plasma rest frame) and the shock size, $L$, as measured along the shock surface, perpendicular to the flow. $L$ is taken equal to the gyroradius of the highest energy shock reflected particle, as measured downstream of the shock. 


\section{References}

[1] Al-Dargazelli S.S., Wolfendale A.W., Śmiałkowski A., Wdowczyk J., 1996, J. Phys. G.: Nucl. Part. Phys., 22, 1825

[2] Beck R., 1997, in Physics of galactic halos, eds. H. Lesh, R.J. Dettmar, U. Mebold and R. Schlickeiser, p. 135, Akademie-Verlag, Berlin

[3] Bednarz J., Ostrowski M., 1998, Phys. Rev. Lett., 80, 3911

[4] Bednarz J., Ostrowski M., 1999, MNRAS, 310, L11

[5] Bertou X., Boratav M., Letessier-Selvon A., 2000, Int. J. Mod. Phys. A15, 2181

[6] Blasi P., Epstein R.I., Olinto A.V., 2000, ApJ, 533, 123

[7] Boldt E., Gosh P., 1999, MNRAS, 307, 491

[8] Chyży K., Beck R., Hohle S., Klein U., Urbanik M., 2000, A\&A, 355, 128

[9] Drury L.O'C., 1983, Rep. Prog. Phys., 46, 973

[10] Gallant Y.A, Achterberg A., 1999, MNRAS, 305, L6

[11] Hillas A.M., 1984, ARA\&A, 22, 425

[12] Kang H., Rachen J.P., Biermann P.L., 1997, APh, 7, 181

[13] Kronberg P.P., 2001, in High Energy Gamma-Ray Astronomy, eds. F.A. Aharonian \& H. Völk, APJ Conf. Series

[14] Ostrowski M., 1998, A\&A, 335, 134

[15] Ostrowski M., 2000, MNRAS, 312, 579

[16] Ostrowski M., Siemieniec-Oziȩbło G., 2002, A\&A, 386, 829

[17] Rachen J.P., Biermann P., 1993, A\&A, 272, 161

[18] Sigl G., Lemoine M., Biermann P., 1999, APh, 10, 141

[19] Stecker F.W., 2000, Astroparticle Phys., 14, 207

[20] Uchihori Y., Nagano M., Takeda M., Teshima M., Lloyd-Evans J., Watson A.A., 2000, APh, 13, 151

[21] Vietri M., 1995, ApJ, 453, 883

[22] Venkatesan A., Miller M. Coleman, Olinto A.V., 1997, ApJ, 484, 323

[23] Waxman E., 1995, Phys. Rev. Lett., 75, 386 